\title{
Radiation dose in CT-guided microwave liver tumor ablation
}

\author{
Grzegorz Rosiak1, Joanna Podgórska², Katarzyna Pasicz³, Krzysztof Milczarek, \\ Dariusz Konecki ${ }^{1}$, Olgierd Rowiński ${ }^{1}$
}

\begin{abstract}
'Il Radiology Department, Central University Hospital (UCK), Warsaw Medical University, Warsaw, Poland ${ }^{2}$ Radiology Department 1, Maria Sklodowska-Curie National Research Institute of Oncology, Warsaw, Poland ${ }^{3}$ Medical Physics Department, Maria Sklodowska-Curie National Research Institute of Oncology, Warsaw, Poland
\end{abstract}

Introduction. Ablation is one of most important methods of liver tumor treatment. However, radiation is one of disadvantages of CT-guided procedures including ablation. The purpose of this study is to assess the factors that have impact on radiation doses during $\mathrm{CT}$-guided microwave liver tumor ablation.

Material and methods. Radiation doses of CT-guided liver tumor ablations were collected in 127 patients. They were then compared in terms of number of lesions, lesion size and depth, use of additional localization needles and hydrodissection as well as tumor location.

Results. The median radiation doses of ablations of multiple tumors $\left(2348 \mathrm{mGy}{ }^{*} \mathrm{~cm}\right)$ were significantly higher $(p=0.03)$ than those of single tumors ( $1784 \mathrm{mG} y^{*} \mathrm{~cm}$ ). No statistically significant differences were noted when other factors (lesion size, depth, location, use of localization needles and hydrodissection) were taken into consideration.

Conclusions. The number of lesions is the most important factor in terms of expected radiation doses in CT-guided microwave liver tumor ablations.

Key words: microwave ablation, radiation dose, CT-guided ablation

\section{Introduction}

Thermal ablation is an established method of liver tumor treatment $[1,2]$. It is frequently performed with CT-guidance due to its high spatial resolution as well as the ability to precisely visualize needles and organs [3]. However this method of guidance is associated with radiation that can potentially increase the risk of malignancy $[4,5]$. The risk is low but not negligible and, according to the ALARA concept, the radiation should always be kept as low as reasonably possible. This study is an attempt to estimate those factors affecting radiation doses during CT-guided liver ablation procedures.

\section{Materials and methods}

The institutional bioethical committee waived the need for formal consent due to retrospective nature of this study. 127 consecutive patients (85 males, 42 females) underwent liver tumor ablations between 2018 and 2019; 88 of them had single tumor, while 39 patients had multiple (89) tumors. Among the tumors there were 43 hepatocellular carcinomas (HCCS) and 134 metastases: breast cancer $(n=4)$, neuroendocrine tumors (NET) $(n=4)$, colorectal cancer $(n=126)$. The mean age of the patients was 69 years (range 25-91).

\section{How to cite:}

Rosiak G, Podgórska J, Pasicz K, Milczarek K, Konecki D, Rowiński O. Radiation dose in CT-guided microwave liver tumor ablation. NOWOTWORY J Oncol $2022 ; 72: 16$-19. 
Table I. Radiation doses (dose length product - DLP) in ablations of single vs. multiple tumors

\begin{tabular}{|lcccccccc} 
& $\mathrm{n}$ total & Mean & Standard deviation & Minimum & Median & Maximum & $p$ value \\
\hline DLP for single tumors $\left[\mathrm{mGy}{ }^{*} \mathrm{~cm}\right]$ & 88 & 2377 & 1697 & 450 & 1784 & 7518 & 0.03 \\
\cline { 1 - 2 }$\left[\mathrm{mG}{ }^{*} \mathrm{~cm}\right]$ & 39 & 2333 & 746 & 967 & 2348 & 3839 &
\end{tabular}

All procedures were performed percutaneously with a microwave ablation device (Solero, Angiodynamics, Lantham, NY, USA), under general anesthesia. The ablations were done under CT-guidance using 320 slice Toshiba Aquilion One scanner (Toshiba/Canon, Nasu, Japan). Ultrasound was done just before every procedure to make sure no new lesions were visible and the tumor was still ablatable. Non-enhanced CT was performed at the beginning of every procedure to visualize the tumor. Then 3-slice (quick-check) scans were done during the procedure, every time the needle was advanced into the tumor.

After the ablation needle was removed, a 3-phase CT scan was done to estimate the ablation zone size and location, with a special focus on oncological margins of at least 5-10 mm. The following parameters were used for spiral CT scans: $120 \mathrm{kV}$ and $300 \mathrm{~mA}$ for spiral scans or $50 \mathrm{~mA}$ (quick-check scans). No real-time CT-fluoroscopy was used during the procedures.

In 48 patients who had large tumors ( $>20 \mathrm{~mm}$ ), one or two localization needles were used (Chiba, 21G, Cook, Bloomington, IN, USA). Those needles were placed to mark the borders of the tumors that required multiple ablation sessions. Hydrodissection was performed in 5 patients. A thin (22 G) needle was placed under CT guidance in a narrow (1-3 $\mathrm{mm}$ ) space between the liver and adjacent stomach, colon or kidney. Between 50 and $200 \mathrm{ml}$ of normal saline was then injected to isolate these structures from the heat produced during ablation and to prevent thermal damage to those organs.

Data on radiation doses in terms of dose length product were collected from the dose report generated by the scanner. The effective dose in mSv was calculated by multiplying by a factor of 0.015 [6]. The carcinogenic effect of the procedure, defined as excess risk of malignancy, was calculated at 5\% per sievert [7].

The CT images from the procedures were retrospectively reviewed and the following data were collected: number of lesions, lesion size, number of localization needles inserted, hydrodissection application, lesion depth (from the entry point on the skin), location of the lesion (liver segment).

\section{Statistical analysis}

The Shapiro-Wilk test was used to assess the normality of distribution of the investigated parameters. Differences were tested by the Wilcoxon rank-sum test and Kruskal-Wallis test. Pearson's correlation was used to analyze the association between DLP versus depth and DLP versus diameter. The values $p<0.05$ were considered statistically significant. Sta- tistical analysis was done using R environment (version 3.3.2, The R-Foundation, Austria).

\section{Results}

Ablations of multiple tumors were associated with higher radiation doses than single tumors in terms of DLP. Median DLP (mGy* $\mathrm{cm}$ ) for single tumors was 1784 (range: 450-7518) while for multiple tumors it was 2348 (967-3839) and the difference was statistically significant ( $p=0.03$ ) (tab. I). The median effective doses were calculated at $26.8 \mathrm{mSv}$ and 35.2 $\mathrm{mS} v$ respectively.

There was no statistical significance ( $p=0.23)$ (fig. 1) in DLP increase in patients in whom localization needles were

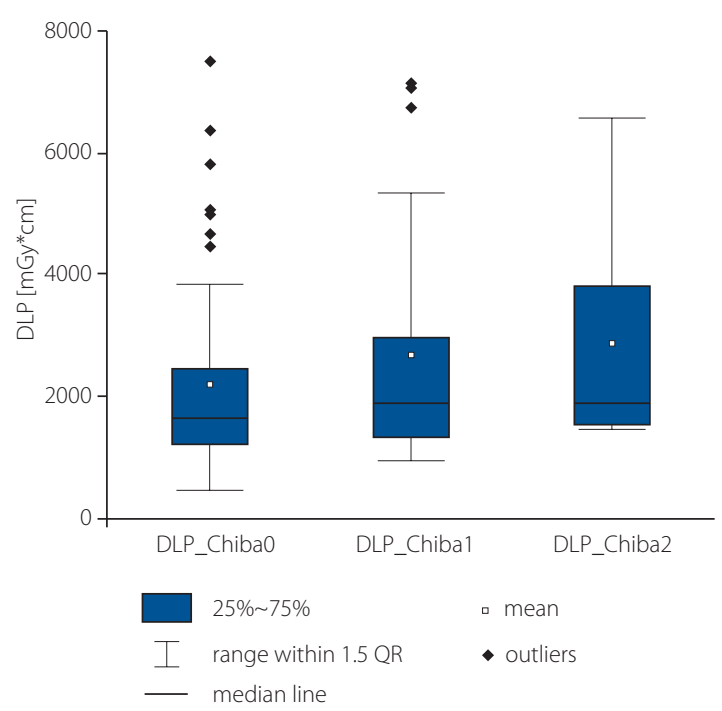

Figure 1. Radiation doses (DLP) by a number of localization needles used

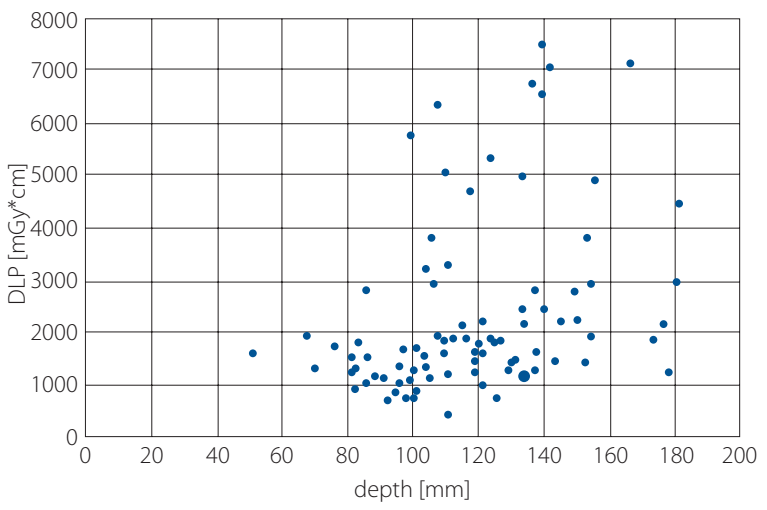

Figure 2. Radiation doses (DLP) by lesion depth 


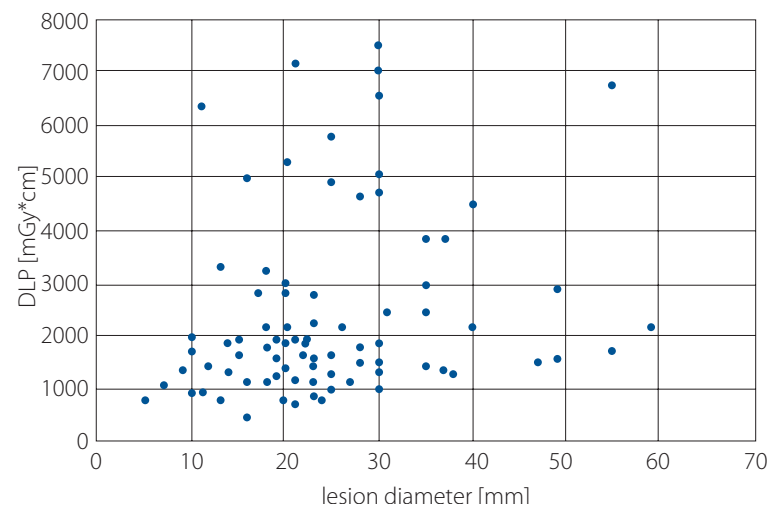

Figure 3. Radiation doses (DLP) by lesion diameter

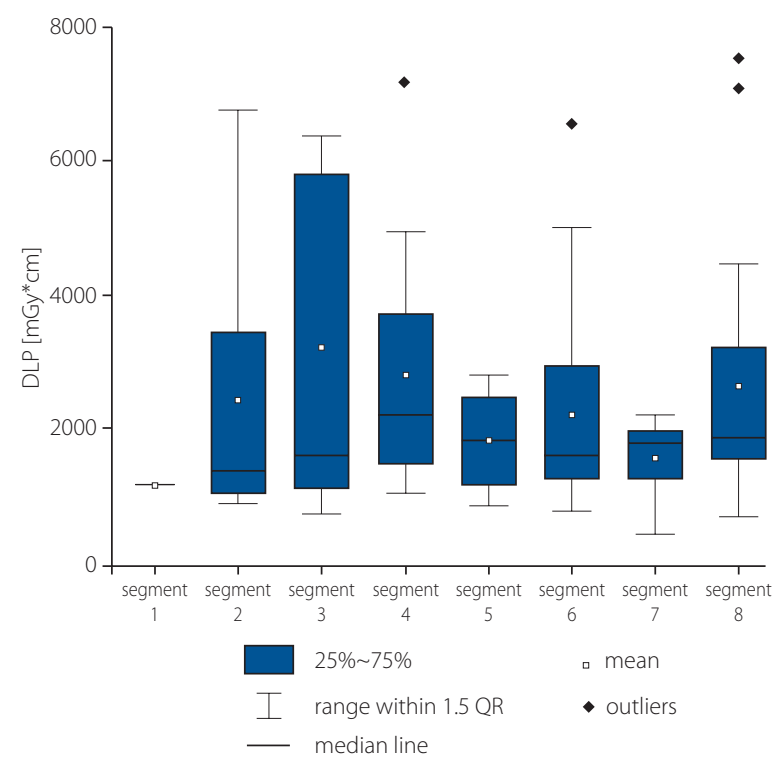

Figure 4. Radiation doses (DLP) in ablations of lesions in particular liver segments

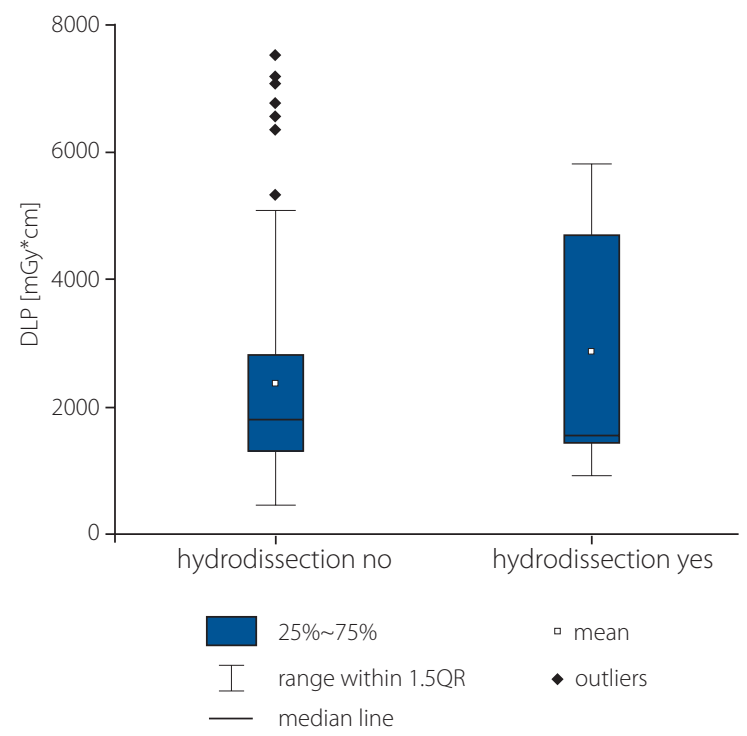

Figure 5. Radiation doses (DLP) during ablation without and with hydrodissection used. The correlation between DLP and lesion depth or size was very weak and was not statistically significant (fig. 2 and fig. 3). Similarly, the location (by liver segment, fig. 4) of the lesion and the use of hydrodissection (fig. 5) did not have a statistically significant impact on the radiation doses. The estimated lifetime excess risk of malignancy was calculated at $0.10 \%$ for ablations of single lesions and $0.14 \%$ for ablations of multiple lesions.

\section{Discussion}

CT-guidance is frequently used in percutaneous liver tumor ablation due to its excellent spatial resolution and ability to visualize organs and needles with high quality. In many cases ultrasound is not able to show all tumors, especially in a cirrhotic liver or after chemotherapy. Additionally, ultrasound is not a reliable way to show the ablation zone and margin size which is an independent predictor of local tumor progression [8].

Radiation is one of the disadvantages of this method and doses should be kept as low as reasonably achievable (ALARA). The radiation doses in terms of DLP had quite a wide range (450-7518 mGy* $\mathrm{cm}$ ). Out of several parameters, the number of ablated lesions was a factor that had a significant impact on the radiation dose. Ablation of multiple tumors caused higher radiation than procedures done on single lesions (median 2348 vs. 1784 mGy* ${ }^{*} \mathrm{~cm}$ which corresponds to 35 vs. 26 mSv).

The results are comparable to other studies. In a publication by $\mathrm{Hu}$ et al. [9] the radiation doses acquired during CT-guided ablation were slightly higher and estimated at $41.1 \mathrm{mSv}$. Similar results were reported by McCarthy et al. [10] where the estimated radiation dose was $30.7 \mathrm{mSv}$. It is worth noting that the results are similar in many aspects even though the procedures were performed in different centers on different $\mathrm{CT}$ scanners.

As opposed to the results of the study by McCarthy et al. [10], hydrodissection was not a factor that would cause a statistically significant increase in radiation dose. The small number of patients that had this additional measure applied in our study could be the reason for such results. However, this result corresponds to other data in our study, especially the application of localization needles as both techniques (hydrodissection and localization needles) require additional punctures and should have a similar impact on the radiation dose.

The lack of statistical significance between radiation doses in the ablation of small and large lesions was somewhat unexpected since large lesions require more needle repositioning and thus more scans. Radiation doses for patients with additional localization needles did not show statistically significant differences. Higher radiation doses in such procedures were expected since they required additional scans to insert the needles precisely into the tumor's border. Moreover, there was a lack of statistical significance when lesion size, depth or location (liver segment) were taken into consideration. 
The range of radiation dose values was fairly wide so it remains possible that factors other than the number of lesions have a significant impact. If the effects of lesion size, depth, location (liver segment), hydrodissection and additional needles on radiation doses exist, they seem to have been dominated by other, unknown factors. The effect of "difficulty" of the procedure could be such a factor. Some tumors are more difficult to ablate than others, but no clear parameters have been defined so far. It is possible that the difficulty of the procedure depends on many factors and such complexity makes it hard to clearly define it. That said, the search for such parameters could be a subject of further studies.

This study did not include contrast injections as a factor potentially affecting the radiation dose [10] since all patients had a contrast enhanced CT after needle removal. This step is necessary to assess margin size which predicts the risk of local tumor progression [11]. The majority of radiation doses in CT-guided procedures comes from helical scans [12]. Limiting such scans by replacing some of them with quick-check scans can significantly reduce the radiation dose in CT-guided procedures [13]. However, it can be difficult in such complex procedures as ablations where the operator needs to have high quality visualizations of large volumes of liver tissue. While limiting radiation in CT-guided procedures is important, it should not be done at the cost of reducing the effectiveness of precise needle placement.

The excess risk of malignancy was calculated at 0.10 (single lesions) or $0.14 \%$ (multiple lesions) which compares favorably with $0.43 \%$ of children and young adults who underwent regular CT scans [14]. The radiation doses acquired by patients who underwent liver tumor ablations correspond to doses acquired during 2-4 multiphase abdominal CT scans.

Liver tumor ablation is a safe procedure with very low major complication rates, from 1.1\% [15] to 5\% [16], with practically no post-procedural mortality. This compares favorably to liver tumor resection where complications tend to be more frequent, e.g. $27.5 \%$ [16]. The results of our study show that excess risk of malignancy in liver tumor ablation is low and in our opinion it should not be a major factor when making decisions on liver tumor treatment. Considering the high efficacy of ablation in liver tumor treatment [17] and its low carcinogenic effect, the potential health gains outweigh the risks of the procedure. The retrospective nature of this study is one of its limitations. Variations in ablation technique between the operators may have also affected the outcomes. Also, the applied conversion factor that was derived from ICRP [7] is designed to estimate the risk to the general population more than individual patients.

\section{Conclusions}

The radiation doses and excess risk of malignancy in CT-guided liver ablation are low. The risks are higher in ablations of multiple tumors, however lesion size, depth and location or application of hydrodissection or additional needles do not have a significant impact on radiation dose.
Conflict of interest: none declared

\author{
Joanna Podgórska \\ Maria Sklodowska-Curie National Research Institute of Oncology \\ Radiology Department 1 \\ ul. Roentgena 5 \\ 02-781 Warszawa, Poland \\ e-mail:jpodgo@gmail.com
}

Received: 7 Sep 2021

Accepted: 30 Nov 2021

\section{References}

1. Benson AIB, D'Angelica MI, Abbott DE, et al. Hepatobiliary Cancers, Version 2.2021, NCCN Clinical Practice Guidelines in Oncology. J Natl Compr Canc Netw. 2021; 19(5): 541-565, doi: 10.6004/jnccn.2021.0022, indexed in Pubmed: 34030131.

2. NCCN. Hepatobiliary cancers (Hepatocelluar) v. 2.2021. NCCN. 2021.

3. Baère Tde. Computed Tomography Imaging for Tumor Ablation. Tumor Ablation. 2005: 104-120, doi: 10.1007/0-387-28674-8 9.

4. Brenner DJ, Elliston CD, Hall EJ, et al. Estimated Risks of Radiation. Am Roentgen Ray Soc. 2001; 176: 289-96.

5. Sodickson A, Baeyens PF, Andriole KP, et al. Recurrent CT, cumulative radiation exposure, and associated radiation-induced cancer risks from CT of adults. Radiology. 2009; 251(1): 175-184, doi: 10.1148/ radiol.2511081296, indexed in Pubmed: 19332852.

6. Coakley FV, Gould R, Yeh BM, et al. CT radiation dose: what can you do right now in your practice? AJR Am J Roentgenol. 2011; 196(3): 619-625, doi: 10.2214/AJR.10.5043, indexed in Pubmed: 21343506.

7. The 2007 Recommendations of the International Commission on Radiological Protection. ICRP publication 103. Ann ICRP. 2007; 37(2-4): 1-332, doi: 10.1016/j.icrp.2007.10.003, indexed in Pubmed: 18082557.

8. Wang X, Sofocleous CT, Erinjeri JP, et al. Margin size is an independent predictor of local tumor progression after ablation of colon cancer liver metastases. Cardiovasc Intervent Radiol. 2013; 36(1): 166-175, doi: 10.1007/s00270-012-0377-1, indexed in Pubmed: 22535243.

9. HuEY, Levesque VM, Bay CP, et al. LiverTumor Ablation Procedure Duration and Estimated Patient Radiation Dose:Comparing Positron Emission Tomography/CT and CT Guidance. J Vasc Interv Radiol. 2020; 31(7): 1052-1059, doi: 10.1016/j.jvir.2019.11.036, indexed in Pubmed: 32534979.

10. McCarthy CJ, Kilcoyne A, Li X, et al. Radiation Dose and Risk Estimates of CT-Guided Percutaneous Liver Ablations and Factors Associated with Dose Reduction. Cardiovasc Intervent Radiol. 2018; 41(12): 1935-1942, doi: 10.1007/s00270-018-2066-1, indexed in Pubmed: 30132100.

11. Crocetti $L$, de Baére T, Pereira PL, et al. CIRSE Standards of Practice on Thermal Ablation of Liver Tumours. Cardiovasc Intervent Radiol. 2020; 43(7): 951-962, doi: 10.1007/s00270-020-02471-z, indexed in Pubmed: 32382856.

12. Sarti M, Brehmer WP, Gay SB. Low-dose techniques in CT-guided interventions. Radiographics. 2012; 32(4): 1109-19; discussion 1119, doi: 10.1148/rg.324115072, indexed in Pubmed: 22786997.

13. Rosiak G, Lusakowska A, MilczarekK, et al. Ultra-low radiation dose protocol for CT-guided intrathecal nusinersen injections for patients with spinal muscular atrophy and severe scoliosis. Neuroradiology. 2021;63(4):539-545, doi: 10.1007/s00234-021-02643-9, indexed in Pubmed: 33512541.

14. Bosch de Basea M, Moriña D, Figuerola J, et al. Subtle excess in lifetime cancer risk related to $\mathrm{CT}$ scanning in Spanish young people. Environ Int. 2018; 120: 1-10, doi: 10.1016/j.envint.2018.07.020, indexed in Pubmed: 30053755.

15. Liu Y, Li S, Wan X, et al. Efficacy and safety of thermal ablation in patients with liver metastases. Eur J Gastroenterol Hepatol. 2013; 25(4): 442-446, doi: 10.1097/MEG.0b013e32835cb566, indexed in Pubmed: 23470267.

16. Fang $Y, C$ Chen $W$, Liang $X$, et al. Comparison of long-term effectiveness and complications of radiofrequency ablation with hepatectomy for small hepatocellular carcinoma. J Gastroenterol Hepatol. 2014; 29(1): 193-200, doi: 10.1111/jgh.12441, indexed in Pubmed: 24224779.

17. Ruers T, Van Coevorden F, Punt CJA, et al. European Organisation for Research and Treatment of Cancer (EORTC), Gastro-Intestinal Tract Cancer Group, Arbeitsgruppe Lebermetastasen und tumoren in der Chirurgischen Arbeitsgemeinschaft Onkologie (ALM-CAO), National Cancer Research Institute Colorectal Clinical Study Group (NCRI CCSG). Local Treatment of Unresectable Colorectal Liver Metastases: Results of a Randomized Phase II Trial. J Natl Cancer Inst. 2017; 109(9), doi: 10.1093/jnci/djx015, indexed in Pubmed: 28376151. 